\title{
Transient MHD Couette Flow in a Rotating Environment Permeated by an Inclined Magnetic Field by Means of a Traveling Magnetic Field Subject to a Forced Oscillation
}

\author{
Swapan Kumar Ghosh \\ Department of Mathematics, Narajole Raj College, Narajole, West Bengal, India \\ Email: g_swapan2002@yahoo.com
}

How to cite this paper: Ghosh, S.K. (2018) Transient MHD Couette Flow in a Rotating Environment Permeated by an Inclined Magnetic Field by Means of a Traveling Magnetic Field Subject to a Forced Oscillation. World Journal of Mechanics, 8, 362-377.

https://doi.org/10.4236/wjm.2018.89027

Received: August 17, 2018

Accepted: September 15, 2018

Published: September 18, 2018

Copyright $\odot 2018$ by author and Scientific Research Publishing Inc. This work is licensed under the Creative Commons Attribution International License (CC BY 4.0).

http://creativecommons.org/licenses/by/4.0/

\begin{abstract}
The present investigation reveals to a magnetohydrodynamic (MHD) start up flow of a rotating environment permeated by a traveling magnetic field with reference to a periodic driving force to explore the behaviour of a magnetic field at the resonant level. In a time varying electromagnetic field of sinusoidal in nature subject to $\nabla \cdot J \neq 0$, an oscillating current flow emerges the backbone of a radio emission in which the emission of hot electron gyrates in a magnetic field in the presence of a radiofrequency accelerator. The expedition of a radiofrequency field determines $\mathrm{X}$-emission to expedite radiofrequency voltage under the influence of A.C circuit subject to $\nabla \cdot J \neq 0$. An oscillating current flow deals with an excitation frequency in such a way that phase angle rotates with angular frequency, the magnetic field grows towards the resonant level when the phase angle $\omega \tau=\pi / 2$ is compatible with $\omega>0$. In turn, plasma induced laser radiation is influenced by an oscillator with a decisive importance to an excitation frequency in the presence of a magnetic mirror so that maximum reflection occurs as the magnetic field increase in strength abruptly to exhibit resonance fluorescence. A synchronized laser photon light in transmitted from the Sun subject to $\omega \tau=\pi / 2$.
\end{abstract}

\section{Keywords}

MHD Start Up Flow, Forced Oscillation, Stagnation, Radio Emission, X-Emission, Resonance Fluorescence, Laser Radiation

\section{Introduction}

In recent year, MHD Couette flow in a rotating system has received wide atten- 
tion in literature. A literature survey reveals to the study of Ghosh [1] with a view to analyze the effects of Hall current on MHD Couette flow in a rotating system with arbitrary magnetic field. Guria et al. [2] studied an unsteady MHD Couette flow in a rotating system. Several studies have been made by the researchers with different conditions and configurations. Mention may be made on their works of Pai [3], Mondal et al. [4], Mondal and Mandal [5], Katagiri [6], Muhuri [7], Ghosh and Pop [8], Ghosh [9], Sengupta and Ray [10], Singh [11], Singh and Sharma [12]. Nevertheless, an MHD flow in a rotating system subject to a forced oscillation has been studied by Ghosh [13] and Ghosh et al. [14]. The aim of present investigation is to deal with the configuration of the Sun in the presence of a radiofrequency accelerator. Sun is an MHD in the presence of a radiofrequency accelerator permeated by a magnetic mirror. The configuration of the Sun leads to vacuum in the presence of radiofrequency accelerator so that frictional layer at the boundary suddenly sets into motion leading to a start up flow. It reveals that in space, pressure is far below normal atmospheric pressure and the flow is oscillating in nature with reference to a driving force. A magnetic mirror with the Sun represents a controlled thermonuclear fusion reaction to expedite an MHD of the Sun. In this situation, a magnetic mirror is the representation of an inclined magnetic field. Since forced oscillation is taken into account, a physical system is acted upon by an external periodic driving force in which the resulting amplitude of oscillation of the system becomes large when the frequency of the driving force approaches a natural frequency of oscillation of the system. In turn, internal energy is converted into kinetic energy during convection process and a maximum dissipation of energy is liberated from the Sun with large amplitude when kinetic energy is transformed into heat. The growing magnetic field at the resonance level leads to a driving force in the presence of a magnetic mirror. Although a radiofrequncy accelator generates a radio wave propogation into the atmosphere to expedite oceanic circulation with reference to a magnetic mirror at the resonant level. An expedition of a radio wave in a vacuum emerges the exploration of atmospheric science which is visible in the Earth's atmosphere. This situation reveals that light wave is transmitted in the form of a radio wave with a decisive importance to a controlled thermonuclear fusion reaction of the Sun. To increase radiofrequency voltage, the emission of hot electron gyrates in a magnetic field to exhibit radio emission with a synchronization of $\mathrm{X}$-emission so that magnetic field oscillates in a vacuum. In turn, X-emission determines an irregular fluctuation of a magnetic field in a vacuum. Sun is a MHD with a decisive importance to a magnetic mirror where plasma fusion takes place in a magnetic field at the resonant level with reference to a controlled thermonuclear fusion reaction. An oscillator takes place of a driving force in the presence of a magnetic mirror with the Sun exerts its influence of a plasma induced laser radiation so that the system rotates with angular frequency with the growing of a magnetic field towards the resonant level and the angular frequency is determined by the phase angle 
subject to a periodic driving force. The situation reveals that the magnetic field increases in strength abruptly in the presence of a magnetic mirror and the reflection occurs with an abrupt increase in magnetic field to exhibit resonance fluorescence when phase angle is $\pi / 2$. This indicates that a synchronized photon light is transmitted from the Sun. A radiofrequency accelerator with the Sun corresponds to the differential rotation of the Sun; an irregular fluctuation of hot electron is liberated from the Sun with an increase in differential rotation subject to a cyclonic turbulence so that magnetic field changes its direction abruptly from the central region. Since forced oscillation is taken into account, the differential rotation of the Sun subject to a periodic driving force maintains a similar rotation of the Earth that may cause an effect of solar eclipse.

The aim of present investigation is to study of an MHD start up flow when the frictional layer is suddenly sets into motion. Since the flow is induced by a forced oscillation its motion is subjected to an external periodic driving force which oscillates harmonically with time. MHD start up flow in a rotating environment is influenced by an inclined magnetic field with the positive direction of the axis of rotation. Since the motion is sinusoidal in nature, there arise a fluctuation at every point on the flow field with reference to $\nabla \cdot J \neq 0$ due to the existance of a displacement current. An A.C circuit is furnished under electrical relay analogy to expedite an inclined magnetic field that leads to a radiofrequency voltage and the magnetic field grows towards the resonant level. A strong ionizing radiation inside the solar atmosphere becomes predominant.

\section{Formulation of the Problem and Its Solutions}

A transient hydromagnetic (MHD) start up flow of a viscous incompressible electrically conducting fluid confined between parallel plates lying at a distance " $d$ " apart, rotating with an uniform angular velocity $\Omega$ about an axis perpendicular to their planes in the presence of an inclined magnetic field with the positive directionof the axis of rotation with reference to a forced oscillation permeated by an oscillator, is studied. To choose the cartesian co-ordinate system in such a way that the upper plate is moving with an uniform velocity $U$ relative to the rotating frame of reference while the lower plate is kept fixed so that $x$ and $z$ axis lying on the lower plate and $y$-axis is perpendicular to it. Since the plates are infinite along $x$ and $z$ direction, all physical quantities will be functions of $y$ and $\tau$ only.

The following justifications are compatible with the fundamental equations of magnetohydrodynamics

$$
\begin{aligned}
& q=\left(u^{\prime}, 0, w^{\prime}\right) ; B=\left(B_{x}^{\prime}+B_{0} \sin \theta, B_{0} \cos \theta, B_{z}^{\prime}\right) \\
& E=\left(E_{x}, E_{y}, E_{z}\right) ; J=\left(J_{x}, J_{y}, J_{z}\right)
\end{aligned}
$$

where $q, B, E$ and $J$ are respectively the velocity vector, magnetic field vector, electric field vector and the current density vector.

The MHD momentum equation in a rotating frame of reference reads 


$$
\frac{\partial q}{\partial t}+(q \cdot \nabla) q+2 \Omega \hat{k} \times q=v \nabla^{2} q+\frac{1}{\rho} J \times B
$$

The equation of continuity becomes

$$
\nabla \cdot q=0
$$

The Ohm's law for a moving conductor

$$
J=\sigma[E+q \times B]
$$

The Maxwell's equations are

$$
\begin{aligned}
& \nabla \times B=\mu_{e} J \\
& \nabla \times E=-\frac{\partial B}{\partial t} \\
& \nabla \cdot B=0 \\
& \nabla \cdot J \neq 0
\end{aligned}
$$

where, $\sigma, v, \rho, \mu_{e}, \Omega$ and $\hat{k}$ are, respectively, the electrical conductivity, kinematic coefficient of viscosity, fluid density, magnetic permeability, angular velocity and a unit-vector along y-axis. $B_{0}$ is the magnetic flux density and $\theta$ is the angle of inclination of a magnetic field with the positive direction of the axis of rotation.

In general, the electric current flowing in the fluid distorts the applied magnetic field. Since the frictional layer at the boundary is suddenly sets into motion to produce thermally ionized air which is the best of poor electrical conductor, it is permissible to neglect the induced magnetic field as compared to applied one (Pai [3]). Thus the magnetic field vector can be taken as $B=\left(B_{0} \sin \theta, B_{0} \cos \theta, 0\right)$. Since no external electric field is applied, the polarization voltage is negligible. Therefore, it is reasonably assumed as $E=0$ (Meyer [15]).

Under the justification (1) the MHD momentum equations in a rotating frame of reference subject to a component form together with the Ohm's law for a moving conductor (4) in relation with the Maxwell's Equation (5) can be written in the following form

$$
\begin{gathered}
\frac{\partial u^{\prime}}{\partial t}+2 \Omega w^{\prime}=v \frac{\partial^{2} u^{\prime}}{\partial y^{2}}-\frac{\sigma B_{0}^{2}}{\rho} u^{\prime} \cos ^{2} \theta \\
\frac{\partial w^{\prime}}{\partial t}-2 \Omega u^{\prime}=v \frac{\partial^{2} w^{\prime}}{\partial y^{2}}-\frac{\sigma B_{0}^{2}}{\rho} w^{\prime}
\end{gathered}
$$

The boundary conditions are

$$
\begin{aligned}
& u^{\prime}=w^{\prime}=0 \text { for } t \leq 0,0 \leq y \leq d, \\
& u^{\prime}=w^{\prime}=0 \text { at } y=0, t>0, \\
& u^{\prime}=U, w^{\prime}=0 \text { at } y=d, t>0 .
\end{aligned}
$$

Equations ((6) and (7)) can be written in a dimensionless form such as

$$
\frac{\partial^{2} u}{\partial \eta^{2}}-\frac{\partial u}{\partial \tau}-M^{2} u \cos ^{2} \theta=2 K^{2} w
$$




$$
\frac{\partial^{2} w}{\partial \eta^{2}}-\frac{\partial w}{\partial \tau}-M^{2} w=-2 K^{2} u
$$

where $\eta=\frac{y}{d}, u=\frac{u^{\prime}}{U}, w=\frac{w^{\prime}}{U}, t=\frac{d^{2} \tau}{v}$,

$$
M=B_{0} L(\sigma / \rho v)^{1 / 2} \text { is the Hartmann number and }
$$

$K^{2}=\frac{\Omega d^{2}}{v}$ is the rotation parameter which is the reciprocal of Ekman number.

The corresponding boundary conditions are

$$
\begin{aligned}
& u=w=0 \text { for } \tau \leq 0 \text { and } 0 \leq \eta \leq 1, \\
& u=0, w=0 \text { at } \eta=0, \tau>0, \\
& u=F(\tau), w=0 \text { at } \eta=1, \tau>0
\end{aligned}
$$

Since forced oscillation is taken into account, it is reasonably assumed

$$
\begin{aligned}
& u(\eta, \tau)=u_{0}(\eta) \cos \omega \tau \\
& w(\eta, \tau)=w_{0}(\eta) \cos \omega \tau
\end{aligned}
$$

The corresponding boundary conditions

$$
\begin{aligned}
& u=0, w=0 \text { at } \eta=0, \tau>0, \\
& u=\cos \omega \tau, w=0 \text { at } \eta=1, \tau>0 .
\end{aligned}
$$

Equations ((9) and (10)) are in agreement with $\nabla \cdot J \neq 0$ subject to $J=\left(J_{x}, J_{y}, J_{z}\right)$. This study leads to a displacement current effect due to an external periodic driving force subject to a forced oscillation in the presence of an inclined magnetic field with regard to $\nabla \cdot J \neq 0$. When a displacement current is taken into account, it is possible to consider even those situations which involve non-steady current i.e. situation where $\nabla \cdot J \neq 0$. A.C circuit furnish a special case of time varying electromagnetic phenomenon, where all the variations are sinusoidal in character. The action of such circuits with capacitors as circuit elements can be understood on the basis of the displacement current. Indeed, in a time varying electromagnetic field of sinusoidal in nature subject to $\nabla \cdot J \neq 0$, an inclined magnetic field is the representation of a traveling magnetic field.

Using (13), the Equations ((9) and (10)) can be solved subject to the boundary conditions (14) and solutions for the velocity distributions can be written in the following form.

$$
\begin{gathered}
u(\eta, \tau)=\left[\frac{M^{2} \sin ^{2} \theta+i G^{2}}{2 i G^{2}} \frac{\sinh (\alpha-i \beta) \eta}{\sinh (\alpha-i \beta)}\right. \\
\left.-\frac{M^{2} \sin ^{2} \theta-i G^{2}}{2 i G^{2}} \frac{\sinh (\alpha+i \beta) \eta}{\sinh (\alpha+i \beta)}\right] \cos \omega \tau \\
w(\eta, \tau)=\frac{M^{4} \sin ^{4} \theta+G^{4}}{8 i K^{2} G^{2}}\left[\frac{\sinh (\alpha-i \beta) \eta}{\sinh (\alpha-i \beta)}-\frac{\sinh (\alpha+i \beta) \eta}{\sinh (\alpha+i \beta)}\right] \cos \omega \tau
\end{gathered}
$$


where

$$
\begin{aligned}
\alpha, \beta= & \frac{1}{2}\left\{\left[\left\{\left(1+\cos ^{2} \theta\right) M^{2}-2 \omega \tan \omega \tau\right\}^{2}+G^{4}\right]^{1 / 2}\right. \\
& \left. \pm\left\{\left(1+\cos ^{2} \theta\right) M^{2}-2 \omega \tan \omega \tau\right\}\right\}^{1 / 2}
\end{aligned}
$$

and

$$
G^{2}=\left(16 K^{4}-M^{4} \sin ^{4} \theta-4 \omega^{2} \tan ^{2} \omega \tau-4 \omega^{2}\right)^{1 / 2}
$$

Expressions (17) and (18) are in agreement with Ghosh [13].

The condition(18) can be expressed in the following form

$$
\left(16 K^{4}-M^{4} \sin ^{4} \theta-4 \omega^{2} \tan ^{2} \omega \tau-4 \omega^{2}\right)\left\{\begin{array} { l } 
{ > } \\
{ = 0 } \\
{ < }
\end{array} \text { according as } G ^ { 4 } \left\{\begin{array}{l}
> \\
=0 \\
<
\end{array}\right.\right.
$$

There arises three conditions in such a way that

$\omega>\frac{1}{2} \cos \omega \tau\left(16 K^{4}-M^{4} \sin ^{4} \theta\right)^{1 / 2} \quad, \quad \omega<\frac{1}{2} \cos \omega \tau\left(16 K^{4}-M^{4} \sin ^{4} \theta\right)^{1 / 2} \quad$ and $\omega=\frac{1}{2} \cos \omega \tau\left(16 K^{4}-M^{4} \sin ^{4} \theta\right)^{1 / 2}$

In relevance to the condition $\omega>\frac{1}{2} \cos \omega \tau\left(16 K^{4}-M^{4} \sin ^{4} \theta\right)^{1 / 2}$ it is stated that a resonant response occurs with reference to a driving force with a decisive importance to the phase angle $\omega \tau=\pi / 2$ to prove resonance when the excitation frequency $\omega>0$. Therefore, phase angle rotates with angular frequency of oscillation to build up a rapid oscillation of a charged particle and its amplification is very high when $\omega \tau=\pi / 2$. In this situation, magnetic field grows towards the resonance level so that reflection occurs due to the presence of a magnetic mirror as the magnetic field increases in strength abruptly. This indicates that laser radiation is so intense due to the presence of a magnetic mirror at the resonant level. A radiofrequency accelarator with the Sun in the presence of a magnetic mirror leads to a controlled thermonuclear fusion reaction to produce plasma induced laser radiation due to a driving force subject to an oscillator at the resonant level to prove resonance fluorescence when $\omega \tau=\pi / 2$ and a radio wave propagation from the Sun is visible in the sky (see Figure 1).

In a realistic situation, a radiofrequency accelerator with the Sun emerges the frictional layer at the boundary to set into motion so that space pressure is much below the atmospheric pressure as the air breaks down. In such a case the Sun is exposed to a vacuum and X-rays are liberated from the Sun. A synchroton radiation is transmitted from the Sun in a vacuum as the emission of hot electrons gyrating in a magnetic field to determine radio emission. A magnetic mirror with the Sun is the representation of an inclined magnetic field. A strong ionizing radiation inside the solar atmosphere to deal with plasma induced laser radiation at the resonant level. 


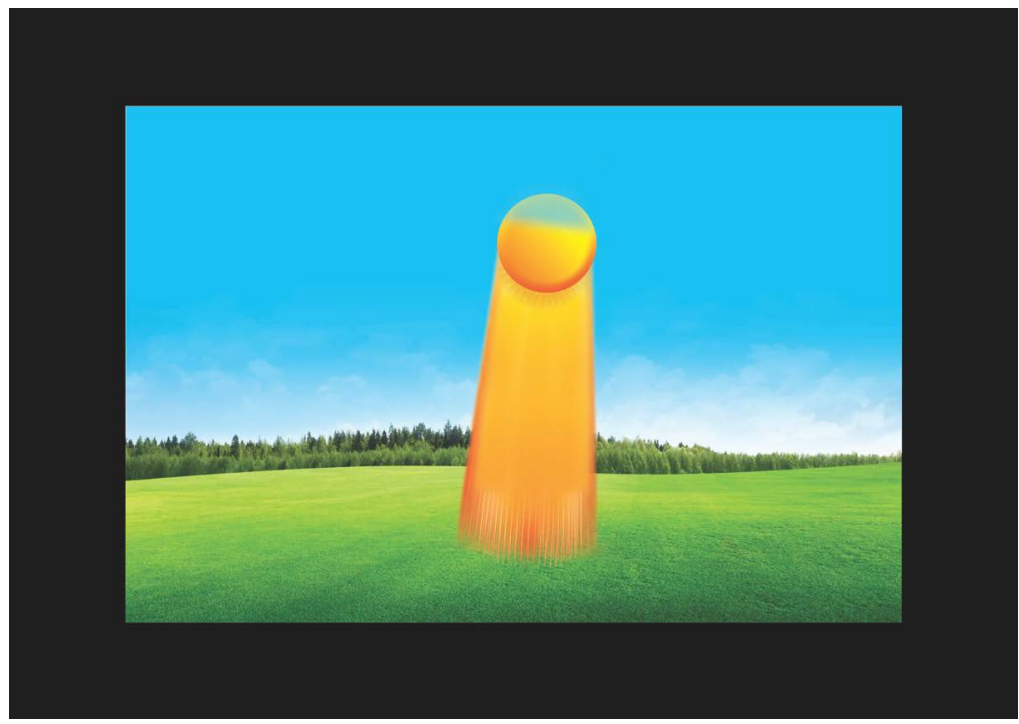

Figure 1. Plasma induced laser radiation shows resonance fluorescence to provide synchronized photon light in the presence of a radiofrequency accelerator under the influence of a magnetic mirror with reference to an oscillator in the context of dynamo mechanism of the Sun. Such a behavior of the Sun was visible in the sky with radio wave propagation in the year of $5^{\text {th }}$ November, 2006.

In compliance with the condition, $\omega<\frac{1}{2} \cos \omega \tau\left(16 K^{4}-M^{4} \sin ^{4} \theta\right)^{1 / 2}$ it is rigorously stated that no resonant response occurs when the phase angle $\omega \tau=\pi / 2$ in accordance with the excitation frequency $\omega<0$.

As referred to a condition $\omega=\frac{1}{2} \cos \omega \tau\left(16 K^{4}-M^{4} \sin ^{4} \theta\right)^{1 / 2}$ there arise stagnation when the phase angle $\omega \tau=\pi / 2$ is compatible with the excitation frequency $\omega=0$. Since phase angle rotates with angular frequency of oscillation, there exists a stagnation so that the velocity will be zero if the excitation frequency is switched off. On the other hand, in the absence of phase angle i.e. $\omega \tau=0$, the condition leads to an inertial frequency $\omega=\frac{1}{2}\left(16 K^{4}-M^{4} \sin ^{4} \theta\right)^{1 / 2}$ in a rotating environment. This justification comes to a conclusion that the Sun is an activation of resonance in the presence of a driving force subject to the resonant condition $\omega>\frac{1}{2} \cos \omega \tau\left(16 K^{4}-M^{4} \sin ^{4} \theta\right)^{1 / 2}$.

The solutions (15) and (16) together with (17) and (18) turn into the general form of a transient MHD start up flow in the presence of an inclined magnetic field if the phase angle is absent $\omega \tau=0$ and the solutions (15) and (16) reduce to

$$
\begin{gathered}
u(\eta, \tau)=\left[\frac{M^{2} \sin ^{2} \theta+i G^{2}}{2 i G^{2}} \frac{\sinh (\alpha-i \beta) \eta}{\sinh (\alpha-i \beta)}-\frac{M^{2} \sin ^{2} \theta-i G^{2}}{2 i G^{2}} \frac{\sinh (\alpha+i \beta) \eta}{\sinh (\alpha+i \beta)}\right] \\
w(\eta, \tau)=\frac{M^{4} \sin ^{4} \theta+G^{4}}{8 i K^{2} G^{2}}\left[\frac{\sinh (\alpha-i \beta) \eta}{\sinh (\alpha-i \beta)}-\frac{\sinh (\alpha+i \beta) \eta}{\sinh (\alpha+i \beta)}\right]
\end{gathered}
$$


where

$$
\alpha, \beta=\frac{1}{2}\left[\left\{\left(1+\cos ^{2} \theta\right)^{2} M^{4}+G^{4}\right\}^{1 / 2} \pm\left(1+\cos ^{2} \theta\right) M^{2}\right]^{1 / 2}
$$

and

$$
G^{2}=\left(16 K^{4}-M^{4} \sin ^{4} \theta-4 \omega^{2}\right)^{1 / 2}
$$

Since phase angle rotates with angular frequency of oscillation, the presence of phase angle comes to a justification in two ways viz,

$\omega>\frac{1}{2} \cos \omega \tau\left(16 K^{4}-M^{4} \sin ^{4} \theta\right)^{1 / 2}$ and $\omega<\frac{1}{2} \cos \omega \tau\left(16 K^{4}-M^{4} \sin ^{4} \theta\right)^{1 / 2}$. The former condition leads to a resonant response with reference to a dynamo mechanism of the system. The later is valid with the maintenance of a magnetic field and the resonant response do not occur. The inertial frequency of a rorating environment does exist when $\omega=\frac{1}{2}\left(16 K^{4}-M^{4} \sin ^{4} \theta\right)^{1 / 2}$.

In taken into account of the solutions (15) and (16) together with (17) and (18) it is stated that since the system rotates with phase angle; if the excitation frequency $\omega$ is switched off then then phase angle $\omega \tau$ is zero and the solutions reduce to a steady state MHD flow in a rotating environment subject to $u(\eta)$ and $w(\eta)$ for (19) and (20) where

$$
\alpha, \beta=\frac{1}{2}\left[\left\{\left(1+\cos ^{2} \theta\right)^{2} M^{4}+G^{4}\right\}^{1 / 2} \pm\left(1+\cos ^{2} \theta\right) M^{2}\right]^{1 / 2}
$$

and

$$
G^{2}=\left(16 K^{4}-M^{4} \sin ^{4} \theta\right)^{1 / 2}
$$

The condition (23) represents a dynamo context of a rotating environment.

\section{Shear Stress at the Moving Plate $\eta=1$}

$$
\begin{aligned}
\left.\frac{\mathrm{d} u}{\mathrm{~d} \eta}\right|_{\eta=1}= & {\left[\frac{M^{2} \sin ^{2} \theta+i G^{2}}{2 i G^{2}} \frac{(\alpha-i \beta) \cosh (\alpha-i \beta)}{\sinh (\alpha-i \beta)}\right.} \\
& \left.-\frac{M^{2} \sin ^{2} \theta-i G^{2}}{2 i G^{2}} \frac{(\alpha+i \beta) \cosh (\alpha+i \beta)}{\sinh (\alpha+i \beta)}\right] \cos \omega \tau \\
\left.\frac{\mathrm{d} w}{\mathrm{~d} \eta}\right|_{\eta=1}= & \frac{M^{4} \sin ^{4} \theta+G^{4}}{8 i K^{2} G^{2}}\left[\frac{(\alpha-i \beta) \cosh (\alpha-i \beta)}{\sinh (\alpha-i \beta)}\right. \\
& \left.-\frac{(\alpha+i \beta) \cosh (\alpha+i \beta)}{\sinh (\alpha+i \beta)}\right] \cos \omega \tau
\end{aligned}
$$

\section{Discussion of Results}

To study of an MHD Start up flow in a rotating system under the influence of a forced oscillation with reference to an inclined magnetic field; a periodic driving force generates an excitation frequency to exhibit resonance subject to $\nabla \cdot J \neq 0$. 
This situation reveals that Ghosh inertal frequency coincides with free natural frequency of oscillation so that oscillation builds up rapidly with a driving force in which the resulting amplitude of oscillation of the system becomes large. Since phase angle rotates with angular frequency, the excitation frequency $\omega>\frac{1}{2} \cos \omega \tau\left(16 K^{4}-M^{4} \sin ^{4} \theta\right)^{1 / 2}$ leads to resonance as the forcing wave to excites natural frequency in compliance with the phase angle $\omega \tau=\frac{\pi}{2}$ with a decisive importance to $\omega>0$. A.C circuit furnishes with a non-steady current flow subject to $\nabla \cdot J \neq 0$ due to a periodic driving force and the growing of a magnetic field at the resonant level in which the emission of hot electron gyrates in a magnetic field to determine radio emission. A charged particle experiences an alternating current flow of sinusoidal in nature with reference to $\nabla \cdot J \neq 0$ that gives rise to radiofrequency voltage under A.C circuit. To increase radiofrequency voltage, the emission of hot electron exerts its influence of $\mathrm{X}$-emission due to irregular fluctuation of a magnetic field in a vacuum. In a time varying electromagnetic field subject to $\nabla \cdot J \neq 0$, an MHD flow is influenced by a traveling magnetic field under the influence of a periodic driving force in the presence of an oscillator. A radiofrequency accelator with the Sun is inspired by a magnetic mirror in the presence of an oscillator. In this context, a controlled thermonuclear fusion reaction at the resonant level so that plasma fusion interacts with the supercritical state as the maximum dissipation of energy is liberated from the Sun with large amplitude when the kinetic energy is transformed into heat and the maximum reflection occurs as the strength of the magnetic field increases its strength abruptly to exhibit plasma induced laser radiation with the propagation of radio wave in the atmosphere.

The graphical discussion in relevance to a physical interpretation has been made with arbitrary values of $\theta, \omega \tau$ and $\omega$ taking $M^{2}$ is fixed in Figures 2-5. Figure 2 reveals that the velocity is exerted by the flow field to increase with an increase the angle of inclination of a magnetic field $\theta$. This happens in the case of a traveling magnetic field in a time varying electrmagnetic field to distort magnetic lines of force due to the presence of Lorentz force with reference to a dynamo machanism of the system. Since the system rotates with angular frequency the velocity is closely resemblance to a resonance when the angle of inclination of a magnetic field $\theta=\pi / 2$. In a time varying electromagnetic field, the magnetic field grows towards the resonant level. In relating to the previous literature, the foundation of the behaviour of an inclined magnetic field in a rotating system does not scope to appear the concept of increasing the angle of inclination of a magnetic field $\theta$. This concept lies on its behaviour of a magnetic field subject to $\nabla \cdot J \neq 0$ at resonance. It is evident from Figure 3 that the velocity increases in the main flow $(u)$ with an increase in magnetic force $\left(M^{2}\right)$ while the velocity decreases in the cross flow $(W)$ with an increase inmagnrtic force $\left(M^{2}\right)$. This situation happens in the case of a main flow $(u)$ to exerts its influence of an angle of inclination of a magnetic field $\theta=\pi / 2$ where the 
Lorentz force is an active influence to distort the magnetic lines of force in the presence of a periodic driving force. In a time varying electromagnetic field, the magnetic field grows subjected to a plasma fusion on heating of plasma in a controlled region at the resonant level when $\theta=\pi / 2$. Thus magnetic pumping in a time varying electromagnetic field becomes predominant. Since the velocity decreases in the cross flow $(w)$, a destabilizing influence on the behaviour of a magnetic pressure leads to fall the velocity due to the presence of Lorentz force when $\theta=\pi / 2$. In general, Lorentz force destabilize the flow situation with an increase in magnetic force. In a radiofrequency field subject to a oscillating current flow in the presence of a forced oscillation, A.C circuit furnishes to

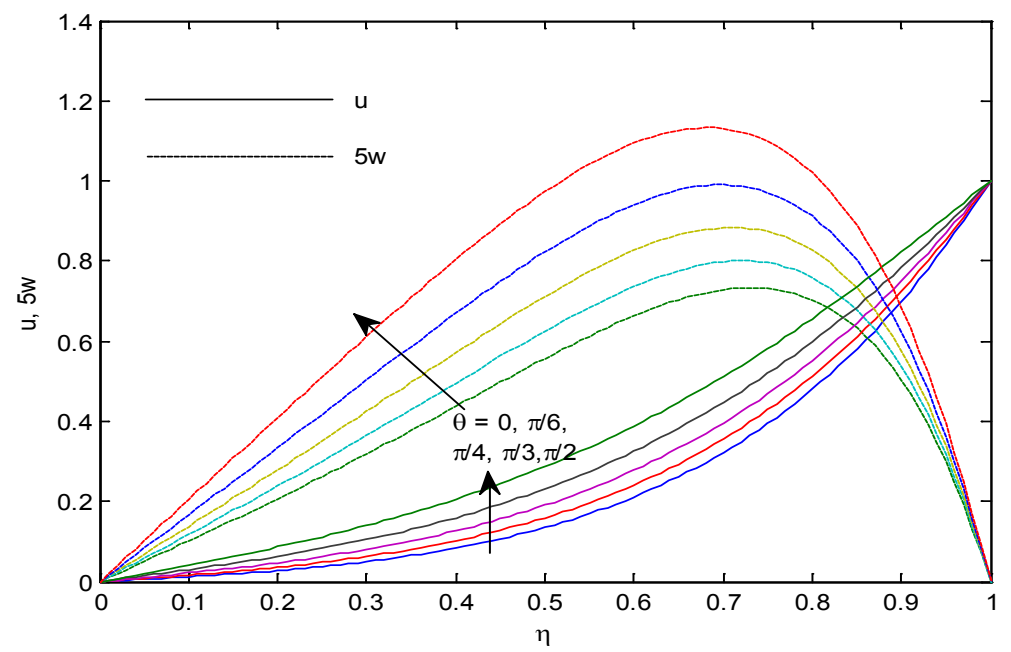

Figure 2. $u$ and $w$ for different values of $\theta=0, \frac{\pi}{6}, \frac{\pi}{4}, \frac{\pi}{3}$ and $\frac{\pi}{2}$ with $M^{2}=10, K^{2}=5, \omega=0.2$ and $\omega \tau=0$.

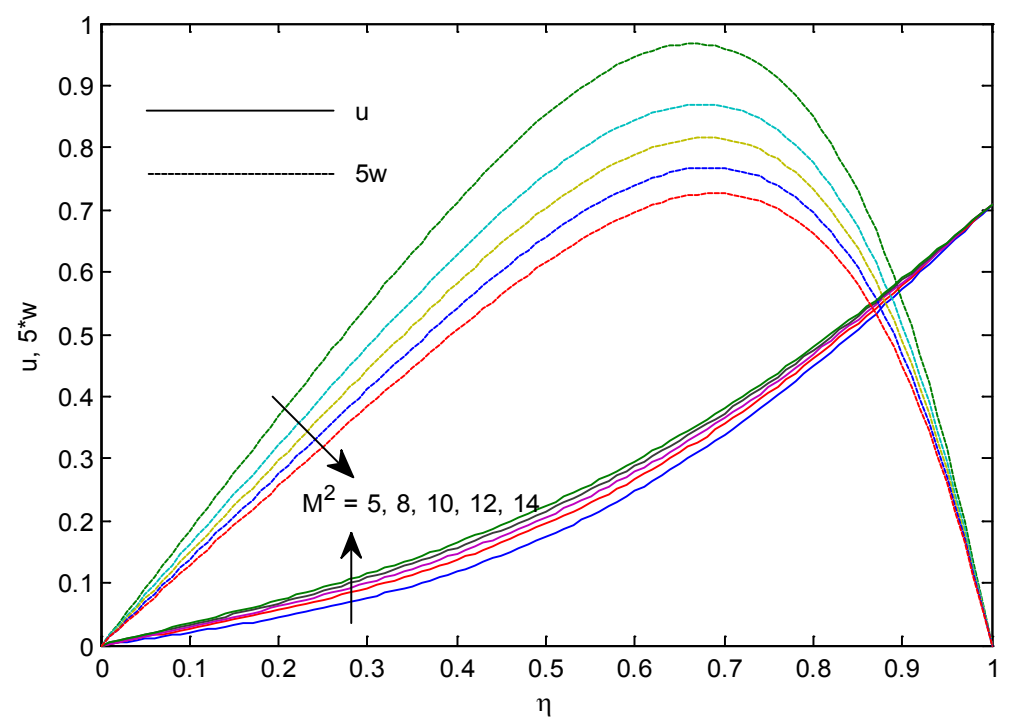

Figure 3. $u$ and $w$ for different values of $M^{2}=5,8,10,12$ and 14 with $K^{2}=5, \omega=0.2, \omega \tau=\frac{\pi}{4}$ and $\theta=\frac{\pi}{2}$. 


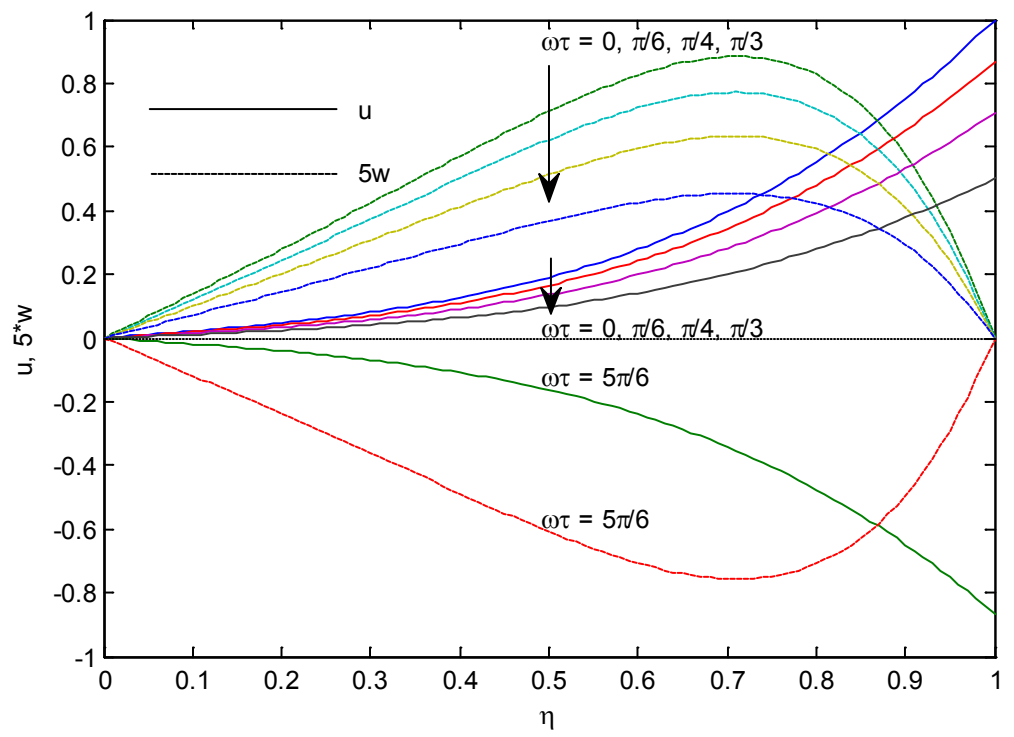

Figure 4. $u$ and $w$ for different values of $\omega \tau=0, \frac{\pi}{6}, \frac{\pi}{4}, \frac{\pi}{3}$ and $\frac{5 \pi}{6}$ with $M^{2}=10, K^{2}=5, \omega=0.2$ and $\theta=\frac{\pi}{4}$.

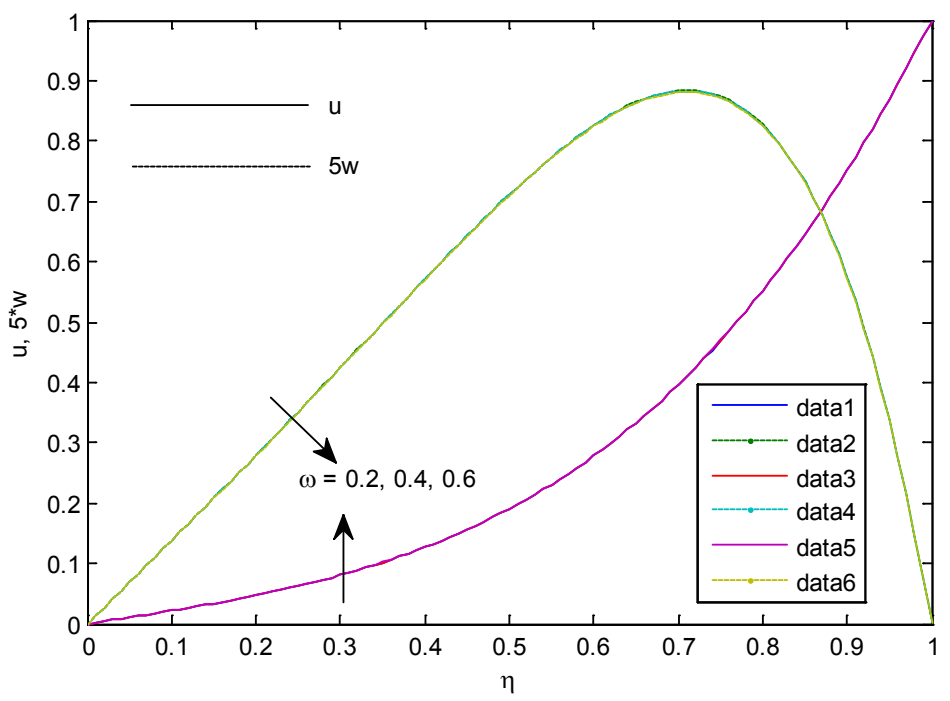

Figure 5. $u$ and $w$ for different values of $\omega=0.2,0.4$ and 0.6 with

$M^{2}=10, K^{2}=5, \omega \tau=0$ and $\theta=\frac{\pi}{4}$.

produce radiofrequency voltage with reference to $\nabla \cdot J \neq 0$ that emerges the backbone of a time varying electromagnetic field to exhibit $\mathrm{X}$-emission in a vacuum. This situation reveals that magnetic pressure increases as the magnetic field grows towards the resonant level due to an irregular fluctuation of a magnetic field. In turn, in a time varying electromagnetic field, these arises fluctuation at every point on the flow field. Figure 4 shows that the velocities due to main flow $(u)$ and cross flow $(w)$ decreases in the range $0 \leq \omega \tau \leq \frac{5 \pi}{12}$ with 
an increase in phase angle $\omega \tau$. Since frictional layer at the boundary is suddenly sets into motion so that air breaks down in a vacuum due to the absence of a pressure gradient. The flow field becomes relevant to the reduction of velocities in the main and cross flows. Although the MHD flow is subjected to a periodic driving force in the absence of a pressure gradient; irregular fluctuation of a velocity field builds up rapidly with a driving force when the phase angle $\omega \tau=\pi / 2$. In a time varying electromagnetic field of a sinusoidal in nature subject to $\nabla \cdot J \neq 0$, the excitation frequency $\omega>\frac{1}{2} \cos \omega \tau\left(16 K^{4}-M^{4} \sin ^{4} \theta\right)^{1 / 2}$ leads to resonance so that irregular fluctuation builds up rapidly with a driving force at large amplitude when $\omega \tau=\pi / 2$. Since forcing wave excites natural frequency the phase angle $\omega \tau=\pi / 2$ is compatible with $\omega>0$. In such a situation, the phase angle $\omega \tau$ rotates with angular frequency $\omega$, the time varying electromagnetic field determines a dynamo context of the Sun. It reveals that in a turbulent regime, a controlled thermonuclear fusion reaction of the Sun becomes significant over a large amplitude subject to a maximum dissipation of energy when the kinetic energy is transformed into heat. This situation implicates the theory of a supercritical state in the presence of a magnetic mirror at the resonant level so that maximum refection occurs with the growing of a magnetic field to exhibit laser radiation as the magnetic field increases in strength abruptly. Indeed an oscillator takes place of a driving force in the presence of a magnetic mirror is subjected to a laser radiation in a time varying electromagnetic field to exert its influence of resonance fluorescence when the phase angle $\omega \tau=\pi / 2$. A synchronized photon light (laser pulse radiation) is transmitted from the Sun with radio wave propagation at shorter wave length. A physical occurrence communicates with the excitation frequency in such a way that irregular changes of galaxies inspired by the Sun due to a differential rotation of the system as the rotation of the Sun maintains a similar rotation of the Earth so that the Sun enters with different galaxies to change of its wave length. It is interesting for the readers that the solar system becomes a steady state in nature if the excitation frequency $\omega$ is switched off so that thermonuclear reaction of the Sun becomes stop. Eventually, the Sun is an exposure of an MHD in the presence of an inclined magnetic field in compliance with resonance subject to a dynamo context of the Sun. Figure 5 demonstrates that the velocity $(u)$ due to main flow increases with an increase in driving frequency $\omega$ whereas the velocity $(w)$ due to cross flow decreases with an increase in driving frequency $\omega$. In a time varying electromagnetic field of a sinusoidal character subject to $\nabla \cdot J \neq 0$, the MHD flow exerted by the driving force to increase the velocity $(u)$ in a radiofrequency field to expedite radiofrequency voltage. In a velocity $(w)$ due to cross flow attains to decrease the velocity to extract radiofrequency voltage in a usual manner as directed by the driving frequency $\omega$.

An illustration takes place of a frictional shear stress to represent a physical interpretation on Tables 1-4 with an arbitrary value of $M^{2}, \theta$ and $\omega \tau$. The 
influence of a frictional shear stress plays a major role at the boundary of the solar region when the frictional layer at the boundary is suddenly sets into motion. Table 1 shows that the frictional shear stress in the main flow $(u)$ direction increases in magnitude with the increase in magnetic pressure $\left(M^{2}\right)$ while it decreases in magnitude with an increase in the angle of inclination $(\theta)$.

Table 1. Frictional shear stress at the moving boundary for $K^{2}=5, \omega=0.2$ and $\omega \tau=0$ due to main flow direction $(u)$.

\begin{tabular}{|c|c|c|c|c|c|}
\hline$\theta$ & 10 & 12 & 14 & 16 & 18 \\
\hline 0 & 3.48092 & 3.72052 & 3.95283 & 4.17731 & 4.39396 \\
\hline$\pi / 6$ & 3.13413 & 3.32258 & 3.50862 & 3.69093 & 3.86880 \\
\hline$\pi / 4$ & 2.75359 & 2.88036 & 3.00984 & 3.14014 & 3.26997 \\
\hline$\pi / 3$ & 2.32825 & 2.37645 & 2.43167 & 2.49213 & 2.55639 \\
\hline$\pi / 2$ & 1.84083 & 1.77992 & 1.72484 & 1.67471 & 1.62881 \\
\hline
\end{tabular}

Table 2. Frictional shear stress at the moving boundary for $K^{2}=5, \theta=\pi / 4$ and $\omega=0.2$ due to main flow direction $(u)$.

\begin{tabular}{|c|c|c|c|c|c|}
\hline$\omega \tau$ & 10 & 12 & 14 & 16 & 18 \\
\hline 0 & 2.75359 & 2.88036 & 3.00984 & 3.14014 & 3.26997 \\
\hline$\pi / 6$ & 2.36981 & 2.47957 & 2.59181 & 2.70483 & 2.81751 \\
\hline$\pi / 4$ & 1.92561 & 2.01520 & 2.10688 & 2.19926 & 2.29139 \\
\hline$\pi / 3$ & 1.34990 & 1.41319 & 1.47806 & 1.54350 & 1.60879 \\
\hline
\end{tabular}

Table 3. Frictional shear stress at the moving boundary for $K^{2}=5, \omega=0.2$ and $\omega \tau=0$ due to cross flow direction $(w)$.

\begin{tabular}{ccccccc}
\hline & \multicolumn{1}{c}{$M^{2}$} & 10 & 12 & 14 & 16 & 18 \\
\hline 0 & & -1.44135 & -1.34654 & -1.26628 & -1.19759 & -1.13816 \\
$\pi / 6$ & -1.51182 & -1.41843 & -1.33823 & -1.26878 & -1.20814 \\
$\pi / 4$ & -1.59681 & -1.50722 & -1.42879 & -1.35979 & -1.29872 \\
$\pi / 3$ & -1.70268 & -1.62177 & -1.54924 & -1.48399 & -1.42509 \\
$\pi / 2$ & -1.84046 & -1.77956 & -1.72449 & -1.67437 & -1.62848 \\
\hline
\end{tabular}

Table 4. Frictional shear stress at the moving boundary for $K^{2}=5, \theta=\pi / 4, \omega=0.2$ due to cross flow direction $(w)$.

\begin{tabular}{|c|c|c|c|c|c|}
\hline$\omega \tau$ & 10 & 12 & 14 & 16 & 18 \\
\hline 0 & -1.59681 & -1.50772 & -1.42879 & -1.35979 & -1.29872 \\
\hline$\pi / 6$ & -1.38911 & -1.31071 & -1.24210 & -1.18176 & -1.12838 \\
\hline$\pi / 4$ & -1.13757 & -1.07308 & -1.01667 & -0.96706 & -0.92320 \\
\hline$\pi / 3$ & -1.80819 & -0.76202 & -0.72165 & -0.68617 & -0.65481 \\
\hline
\end{tabular}


Since viscosity initiates thermal energy in a moving layer the internal energy is converted into a kinetic energy of the system; the magnetic pressure exerted by the main flow direction with reference to a driving frequency to increase viscous shear stress at the moving boundary. The effect of viscous shear stress at the moving layer decreases with increase in $\theta$ (angle of inclination of a magnetc field) for a fixed value of a magnetic pressure $\left(M^{2}\right)$. This happens in the case of a viscous shear where the distortion of a magnetic lines force increase the velocity so that the frictional shear stress decreases at the moving boundary. Table 2 reveals that the frictional shear stress at moving layer due to main flow $(u)$ direction increases in magnitude with an increase in magnetic pressure $\left(M^{2}\right)$ for a fixed value of phase angle $(\omega \tau)$ whereas it decreases in magnitude with an increase in phase angle $(\omega \tau)$ for a fixed value of a magnetic pressure $\left(M^{2}\right)$. Since phase angle rotates with angular frequency, the driving frequency accelerates the viscous shear at the moving layer to increase shear stress at the boundary whereas the viscous shear distabilizes at the moving layer in accordance with phase angle $(\omega \tau)$. Table 3 indicates that the frictional shear stress at the moving boundary due to cross flow $(w)$ direction decreases in magnitude with an increase in magnetic pressure $\left(M^{2}\right)$ while it increases in magnitude with an increase in the angle of inclination of a magnetic field $(\theta)$. Since rotation induces cross flow, the velocity gradient at the moving boundary opposes the viscous stress to decrease in magnitude with an increase in magnetic pressure whereas the effect of velocity gradient leads to increase the frictional shear stress at the moving boundary in accordance with the angle of inclination of magnetic field $(\theta)$. Table 4 demonstrates that the frictional shear stress due to a cross flow $(w)$ direction decreases in magnitude with an increase in either the magnetic pressure $\left(M^{2}\right)$ or the phase angle $(\omega \tau)$. It finds the situation in which the velocity gradient at the moving boundary opposes the effect of frictional shear stress in the cross flow $(w)$ direction. Indeed, the effect of viscous shear stress provides a frictional resistance at the moving boundary to execute thermal resistance. Since the frictional layer at the boundary is suddenly sets into motion the internal energy is converted inton a kinetic energy of the system during convection process and the maximum dissipation of energy is liberated into smallest eddies with large amplitude when the kinetic energy is transformed into heat. This implies to a fact that the convective part of the surface of the Sun leads to a controlled thermonuclear fusion reaction in the presence of a magnetic mirror.

\section{Conclusion}

The purpose of present investigation is to deal with an MHD start up flow in a rotating environment with a decisive importance to a driving force in the presence of an inclined magnetic field by means of a traveling magnetic field. In a time varying electromagnetic field of a sinusoidal in nature subject to $\nabla \cdot J \neq 0$, the MHD flow is associated with a driving force in such a way that the emission of a hot electron is gyrating in a magnetic field to represent radio emission. Since 
the MHD flow exerted by a driving force, the phase angle rotates with angular frequency to exerts its influence of an excitation frequency so that forcing wave excites natural frequency to implicate a resonance when the phase angle $\omega \tau=\pi / 2$ as the phase angle $\omega \tau$ is compatible with $\omega>0$. In turn, magnetic field grows towards the resonant level. Plasma fusion interacts with the supercritical state in the presence of a magnetic mirror with reference to a controlled thermonuclear fusion reaction of the Sun. An oscillator takes place of a driving force to execute plasma induced laser radiation in the presence of a magnetic mirror in which reflection occurs as the strength of the magnetic field increases in strength abruptly to exhibit resonance fluorescence when $\omega \tau=\pi / 2$. This situation reveals that a synchronized photon light (laser pulse radiation) is emitted from the Sun.

\section{Conflicts of Interest}

The author declares no conflicts of interest regarding the publication of this paper.

\section{References}

[1] Ghosh, S.K. (2002) Effects of Hall Current on MHD Couette Flow in a Rotating Environment with Arbitrary Magnetic Field. Czechoslovak Journal of Physics, 52, 51-63. https://doi.org/10.1023/A:1013913730086

[2] Guria, M., Jana, R.N. and Ghosh, S.K. (2006) Unsteady Couette Flow in a Rotating System. The International Journal of Non-Linear Mechanics, 41, 838-843. https://doi.org/10.1016/j.ijnonlinmec.2006.04.010

[3] Pai, S.I. (1962) Magnetohydrodynamics and Plasma Dynamics. Springer-Verlag, Vienna. https://doi.org/10.1007/978-3-7091-8083-9

[4] Mondal, G., Mandal, K.K. and Choudhury, G. (1982) Hall Effects on MHD Plasma Couette Flow in a Rotating System. Journal of the Physical Society of Japan, 51 382-389.

[5] Mondal, G. and Mandal, K.K. (1983) Effect of Hall Current on MHD Couette Flow between Thick Arbitrarily Conducting Plate in a Rotating System. Journal of the Physical Society of Japan, 52, 470-477. https://doi.org/10.1143/JPSJ.52.470

[6] Katagiri, M. (1962) Flow Formation in Couette Motion in Magnetohydrodynamics. Journal of the Physical Society of Japan, 17, 393-396. https://doi.org/10.1143/JPSJ.17.393

[7] Muhuri, P.K. (1963) Flow Formation in Couette Motion in Magnetohydrodynamics with Suction. Journal of the Physical Society of Japan, 18, 1671-1675. https://doi.org/10.1143/JPSJ.18.1671

[8] Ghosh, S.K. and Pop, I. (2004) Hall Effect on MHD Plasma Couette Flow in a Rotating Environment. International Journal of Applied Mechanics and Engineering, 9, 293-305.

[9] Ghosh, S.K. (1993) Unsteady Hydromagnetic Flow in a Rotating Channel with Oscillating Pressure Gradient. Journal of the Physical Society of Japan, 62, 3893-3903. https://doi.org/10.1143/JPSJ.62.3893

[10] Sengupta, R. and Ray, T.K. (1990) Oscillatory Couette Flow of a Viscoelastic Electrically Conducting Fluid through a Porous Medium within a Parallel Plate Channel 
in Presence of a Transverse Magnetic Field in a Rotating System. Archieves of Mechanics, 42, 717-721.

[11] Singh, K.D. (2000) An Oscillatory Hydromagnetic Couette Flow in a Rotating System. Zeitschrift fur Angewandte Mathematik und Mechanik (ZAMM), 80, 429-432. https://doi.org/10.1002/1521-4001(200006)80:6<429::AID-ZAMM429>3.0.CO;2-1

[12] Singh, K.D. and Sharma, R. (2001) MHD Three-Dimensional Couette Flow with Transpiration Cooling. Zeitschrift fur Angewandte Mathematik und Mechanik $(Z A M M), 81,651-720$.

[13] Ghosh, S.K. (2001) A Note on Unsteady Hydromagnetic Flow in a Rotating Channel Permeated by an Inclined Magnetic Field in the Presence of an Oscillator. Czechoslovak Journal of Physics, 51, 799-804. https://doi.org/10.1023/A:1011622416362

[14] Ghosh, S.K., Beg, O.A., Zueco, J. and Prasad, V.R. (2010) Transient Hydromagnetic Flow in a Rotating Channel Permeated by an Inclined Magnetic Field with Magnetic Induction and Maxwell Displacement Current Effects. Zeitschrift fur Angewandte Mathematik und Physik (ZAMP) 61, 147-169.

https://doi.org/10.1007/s00033-009-0006-2

[15] Meyer, R.C. (1958) On Reducing Aerodynamic Heat Transfer Rates by Magnetohydrodynamic Techniques. Journal of the Aerospace Sciences, 25, 561-566.

https://doi.org/10.2514/8.7781 